\title{
Carbon and nitrogen abundances in the coronae of Algol B and other evolved stars: Evidence for CNO-cycle processed material
}

\author{
J. H. M. M. Schmitt and J.-U. Ness
}

\begin{abstract}
Hamburger Sternwarte, Universität Hamburg, Gojenbergsweg 112, 21029 Hamburg, Germany
Received 21 March 2002 / Accepted 10 April 2002

Abstract. Using the $\mathrm{Ly}_{\alpha}$-lines of carbon and nitrogen measured in Chandra Low Energy Transmission Grating Spectrometer (LETGS) spectra we study the coronal abundances of these elements in a sample of late-type stars including dwarf stars, the prototypical eclipsing binary Algol, the single giant $\beta$ Cet, and three RS CVn binaries. In the main sequence stars of our sample the flux ratio $R_{\mathrm{NC}}$ between the Ly ${ }_{\alpha}$-lines of nitrogen and carbon is below unity, while $R_{\mathrm{NC}}$ is found to be $\approx 8.5$ for $\beta$ Cet and $>23.3$ for Algol. These values are more than an order of magnitude larger than expected from a solar abundance plasma in collisional equilibrium regardless of the chosen temperature structure. We therefore interpret the anomalously large $R_{\mathrm{NC}}$-ratios as being due to the exposure of CNO-cycle processed material at the surfaces and in the coronae of Algol and $\beta$ Cet.
\end{abstract}

Key words. stars: abundances - stars: activity - stars: coronae - stars: late type X-rays: stars stars: individual: Algol

\section{Introduction}

One of the most intensely studied binary systems is the bright eclipsing binary Algol (= $\beta$ Per). Already the Chinese noted Algol's brightness changes, but it was Goodricke (1783), who rediscovered its brightness changes, noted their periodicity, and provided the essentially correct interpretation by attributing the brightness change to a second, darker body in the system. In modern language Algol is an eclipsing photometric and spectroscopic binary with well determined system parameters. According to the recent determination of system parameters by Al-Naimiy et al. (1985), Algol contains a B8V primary $\left(M=3.77 M_{\odot}, R=3.07 R_{\odot}\right)$ and a K2III secondary $\left(M=0.82 M_{\odot}, R=3.27 R_{\odot}\right)$. Thus the two stars have almost the same size, but their masses differ by almost a factor of five. The more evolved secondary is lighter than the less evolved primary in seeming conflict with stellar evolution theory which predicts faster evolution for more massive stars. This conflict can only be solved by assuming substantial mass transfer from the present-day secondary to the present-day primary, a suggestion first made by Crawford (1955). Plenty of evidence now exists for mass transfer in Algol-type binaries in the form of gas streams, disk- and related features. The role of mass loss in the evolution of Algol systems is presently not clear; in addition to significant mass being donated

Send offprint requests to: J. H. M. M. Schmitt, e-mail: jschmitt@hs.uni-hamburg.de to the present-day primary, significant mass (and angular momentum) may be lost from the system due to a wind.

Stellar evolution theory predicts that in stars more massive than $\approx 2 M_{\odot}$ the CNO-cycle is the dominant mode of energy generation. In the CNO-cycle the atoms of carbon, nitrogen, and oxygen are used as catalysts to convert hydrogen into helium. Its nuclear equilibrium is such that most of the catalytic nuclei participating in the cycle at any given time are found in the form $\mathrm{N}^{14}$. Therefore one expects to find substantial nitrogen enrichment if the cycle is interrupted by lowering the temperature and CNO-cycled material is subsequently brought up to the surface. Mass transfer in a close binary as in an Algol system is a rather dramatic way to expose nuclearly processed material at the surface. Less dramatic ways to achieve the same result also occur during the regular course of stellar evolution. Once the hydrogen fuel in the stellar core is exhausted nuclear burning moves further and further away from the center; the star responds by expanding and thus starts its giant evolution. As discussed by Iben (1967), the rapidly expanding outer convection zones of giants are in contact with the shell burning zones and may again transport nitrogen-enriched material to the surface.

\section{Previous abundance determinations and the role of $\mathrm{X}$-ray spectroscopy}

These predictions of stellar evolution theory have of course been tested by optical spectroscopists. The principal 
source of information on the photospheric abundances of carbon and nitrogen in cool stars comes from $\mathrm{CH}$ and $\mathrm{CN}$ molecules which have absorption bands in the blue part of the spectrum (cf. Luck 1978; Parthasarathy et al. 1983). The measurements of carbon and nitrogen abundances are made even more difficult if the star is in rapid rotation; further, in Algol systems the much brighter primary star contaminates the spectrum, and useful data can only be obtained during a primary eclipse - if at all. Quantitative abundance determinations require the use of model atmospheres, which predict only relatively small effects in the blue part of the spectrum on varying the carbon and nitrogen abundances so that an accurate model of the atmospheric temperature stratification is required. Finally, a value for the oxygen abundance must be chosen or somehow determined from the data; this is particularly difficult if NLTE-effects are important as maybe the case for supergiants. Despite these difficulties, the secondaries of the Algol systems U Cep and U Sge have been studied by Parthasarathy et al. (1983), who find - relative to iron - a carbon deficiency and nitrogen enhancement as expected from theory. In the atmospheres of giants and supergiants anomalously large N/C-ratios were also reported (Luck 1978; Luck \& Lambert 1981, 1985; Sneden et al. 1986). A summary of carbon/nitrogen abundance determinations is given by McCluskey \& Kondo (1992).

Ultraviolet emission lines provide another means to obtain information on elemental abundances. Böhm-Vitense \& Mena-Werth (1992) use the emission lines from CII, $\mathrm{CIV}$, and NV measured with the IUE satellite, to determine elemental abundances. Their approach heavily relies on the underlying form of the differential emission measure distribution function, which is assumed to have the form $E M=B T^{-\gamma}$, with $T$ denoting temperature. The two constants $B$ and $\gamma$ are found from the C II and C IV emission lines at $1330 \AA$ and $1550 \AA$ respectively, and the abundances of other elements (i.e., nitrogen and silicon) are then computed from a comparison of the observed line flux with those expected from the above power law emission measure distribution. With this method Böhm-Vitense \& Mena-Werth (1992) find N/C-abundances in general agreement with photospherically derived abundances, but an inspection of their Table 4 reveals differences of up to $\Delta \log (\mathrm{N} / \mathrm{C})=0.79$ for individual stars.

X-ray spectroscopy opens up a new window to determine elemental abundances and to independently test the above described evolutionary scenarios. The specific advantage of X-ray lines is that they come directly from the atomic species, not from molecules with their sometimes rather complicated chemistry. Further, no assumptions on the abundance of oxygen have to be made. Here we will pursue an approach even independent of the coronal temperature stratification and only relying on hydrogen-like lines, the atomic physics of which appears to be well understood. We stress that the results derived in this paper do not assume a power-law emission measure distribution, and thus the only assumptions entering our abundance
Table 1. Measured line fluxes and $1 \sigma$ errors for the $\mathrm{N}$ and $\mathrm{C} \mathrm{Ly}{ }_{\alpha}$-lines and their flux ratio $R_{\mathrm{NC}}$, where effective areas by (Pease et al. 2000) were used.

\begin{tabular}{rccc}
\hline \hline & N VII & C VI & $R_{\mathrm{NC}}$ \\
\hline$\lambda / \AA$ & 24.74 & 33.74 & $\left(\frac{\mathrm{N} \mathrm{VII}}{C}\right) \times$ \\
$A_{\text {eff }} / \mathrm{cm}^{2}$ & 15.26 & 11.59 & $\frac{\left(A_{\text {eff }} \lambda\right)_{\mathrm{C}_{\mathrm{VI}}}}{\left(A_{\text {eff }} \lambda\right)_{\mathrm{N}_{\mathrm{VII}}}}$ \\
\hline Procyon & $206.9 \pm 16.95$ & $697.5 \pm 28.1$ & $0.31 \pm 0.03$ \\
$\pi^{1}$ UMa & $17.9 \pm 80$ & $14.78 \pm 80$ & $1.25 \pm 0.90$ \\
$\alpha$ Cen A & $17.67 \pm 11.44$ & $117.65 \pm 4.96$ & $0.16 \pm 0.10$ \\
$\alpha$ Cen B & $32.07 \pm 6.89$ & $102.86 \pm 11.26$ & $0.32 \pm 0.08$ \\
$\epsilon$ Eri & $178.85 \pm 15.85$ & $289.88 \pm 18.66$ & $0.64 \pm 0.07$ \\
YY Gem & $91.8 \pm 11.5$ & $138.4 \pm 13.3$ & $0.69 \pm 0.11$ \\
AD Leo & $146.35 \pm 14.41$ & $203.38 \pm 15.54$ & $0.74 \pm 0.12$ \\
\hline HR 1099 & $501.93 \pm 29.32$ & $627.51 \pm 29.62$ & $0.83 \pm 0.06$ \\
Capella & $2280.3 \pm 53.6$ & $2151.1 \pm 51.3$ & $1.10 \pm 0.04$ \\
UX Ari & $602.85 \pm 27.97$ & $309.36 \pm 20.95$ & $2.02 \pm 0.17$ \\
$\beta$ Cet & $200.53 \pm 16.5$ & $24.48 \pm 6.72$ & $8.48 \pm 2.44$ \\
Algol & $1119.05 \pm 38.38$ & $<50$ & $>23.3$ \\
\hline
\end{tabular}

studies are the ionization equilibrium for carbon and nitrogen and the excitation rates for those lines.

The secondaries of Algol systems are - almost per definitionem - rapidly rotating and hence active late type stars. Most single giants in a volume-limited sample around the Sun are found to be X-ray sources (cf. Schröder et al. 1998), and only for a few stars really sensitive low upper limits to the absence of any X-ray emission have been established (cf. Ayres et al. 1991). Therefore for the secondaries of Algol systems as well as single giants X-ray emission is the rule rather than the exception. This X-ray emission is produced by plasma in collisional equilibrium at temperatures where carbon, nitrogen, and oxygen are predominantly found as helium-like and hydrogen-like ions. The strongest line from hydrogenlike ions is the $\mathrm{Ly}_{\alpha}$-line, from helium-like ions it is the resonance line ${ }^{1} \mathrm{~S}_{0}-{ }^{1} \mathrm{P}_{1}$ in the triplet transition array. The $\mathrm{Ly}_{\alpha}$ lines of carbon, nitrogen, and oxygen are located at $34.74 \AA, 24.74 \AA$, and $18.97 \AA$, and the helium-like resonance lines at $40.3 \AA, 28.7 \AA$, and $21.6 \AA$ respectively. They can therefore be perfectly observed with the LETGS onboard Chandra.

\section{Observations and data analysis}

The LETGS onboard Chandra covers the spectral range between $6 \AA-170 \AA$ (i.e., including the spectral range covered with the ROSAT PSPC) with a spectral resolution of $\approx 0.03 \AA$; a detailed description of this instrument is given by Predehl et al. (1997). In Fig. 1 we show portions of the accumulated LETGS spectra covering the O VII triplet and the nitrogen $\mathrm{Ly}_{\alpha}$-line (left panel) and the carbon $\mathrm{Ly}_{\alpha}$-line (right panel) for our sample stars Algol (= $\beta$ Per $)$, the single giant $\beta$ Cet (spectral type K0III), the RS CVn binary UX Ari (spectral type G5V+K0V), and 

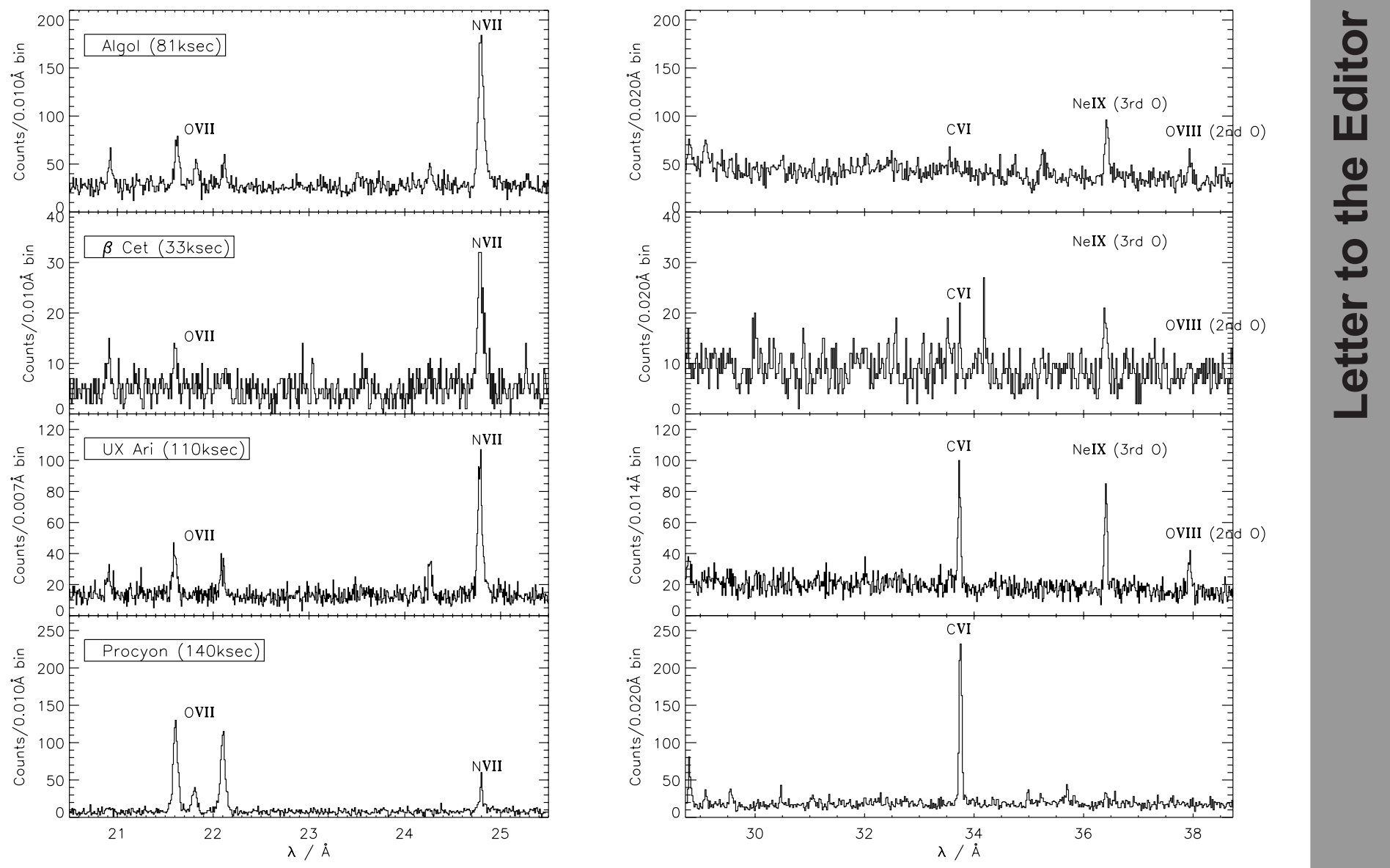

Fig. 1. LETGS spectra (background subtracted) of Algol, $\beta$ Cet, UX Ari, and Procyon in the spectral regions $21-25 \AA$ covering the $\mathrm{O}$ VII triplet and the $\mathrm{N}$ VII $\mathrm{Ly}_{\alpha}$-line as well as $29-39 \AA$ covering the $\mathrm{C}_{\mathrm{VI}} \mathrm{Ly}_{\alpha}$-line; note the different relative strengths of the $\mathrm{N}$ and $\mathrm{C} \mathrm{Ly}_{\alpha}$-lines in the sample stars.

the nearby subgiant star Procyon (spectral type F5IV-V); the LETGS spectra of Procyon, Algol, and YY Gem have been discussed by Ness et al. (2001), Ness et al. (2002), and by Stelzer et al. (1998), respectively. The O VII triplet and the nitrogen $\mathrm{Ly}_{\alpha}$-line are seen in all stars, while the strength of the carbon $\mathrm{Ly}_{\alpha}$-line varies considerably. In Procyon the strength of carbon $\mathrm{Ly}_{\alpha}$ exceeds that of nitrogen $\mathrm{Ly}_{\alpha}$, in Algol the carbon $\mathrm{Ly}_{\alpha}$-line cannot be detected. In Table 1 we list the observed number of counts (and their errors) in the carbon and nitrogen $\mathrm{Ly}_{\alpha}$-lines and their flux ratio $R_{\mathrm{NC}}$; the line strengths were determined with the same methods as discussed by Ness et al. (2001). In addition to the stars shown in Fig. 1 we included Capella (spectral type G1III+G8/K0III) and HR 1099 (spectral type K1IV+G5IV), two RS CVn binaries, and the dwarf stars $\epsilon$ Eri (spectral type K2V), $\alpha$ Cen A (spectral type G2V) and B (spectral type K0V), YY Gem (spectral type dM1e+dM1e), $\pi^{1}$ UMa (spectral type G1.5V), and $\mathrm{AD}$ Leo (spectral type dM4.5Ve). In Fig. 2 we plot our measurements and a theoretical curve (solid line) of the expected ratio $R_{\mathrm{NC}}$ of the nitrogen and carbon $\mathrm{Ly}_{\alpha^{-}}$ line fluxes as a function of plasma temperature (solid line in Fig. 2 as calculated by Mewe et al. (1985) under the assumption of collisional equilibrium and cosmic abundances). Under those assumptions one expects $R_{\mathrm{NC}} \leq 0.57$ independent of the actual plasma temperature (cf., Fig. 2). Since the precise plasma temperature is not known, and in fact a whole distribution of plasma at different temperatures is likely to be present in stellar coronae, the true value of $R_{\mathrm{NC}}$ should be $<0.57$.

\section{Results, discussion and conclusions}

An inspection of Fig. 2 and the corresponding measurements given in Table 1 shows for the stars $\epsilon$ Eri, $\alpha$ Cen A and B, YY Gem, $\pi^{1} \mathrm{UMa}$, and AD Leo that the measured values of $R_{\mathrm{NC}}$ are consistent (to within the measurement errors) with the theoretical expectations from a cosmic abundance plasma in collisional equilibrium. However, the giant stars Capella, HR 1099, UX Ari, $\beta$ Cet, and Algol lie - in increasing strength - significantly above the theoretical expectations. For the latter no emission from $\mathrm{C}$ VI (or any other carbon ion) has been measured so that only a lower limit $\left(R_{\mathrm{NC}, \mathrm{Algol}} \geq 23.3\right)$ can be derived, and the value measured for $\beta$ Cet $\left(R_{\mathrm{NC}, \beta \text { Cet }}=8.48 \pm 2.4\right)$ deviates by more than one order of magnitude from theoretical expectations. Thus all giants in our sample show significantly enhanced $R_{\mathrm{NC}}$-ratios, while all dwarf stars have 


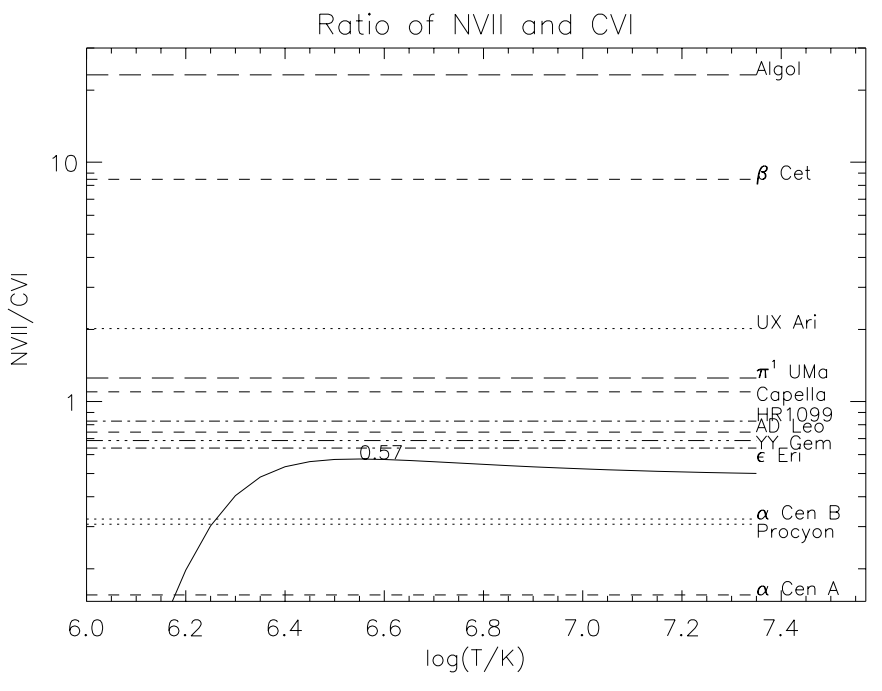

Fig. 2. LETGS flux ratios of N vil and C VI for twelve stars compared to MEKAL flux ratio vs. temperature $T$ (solid line). Algol, $\beta$ Cet, UX Ari, Capella, and HD 1099 lie significantly above the MEKAL curve for any temperature; the other stars are consistent with the MEKAL curve.

$R_{\mathrm{NC}}$-ratios consistent with cosmic abundances. We propose to attribute these anomalously large $R_{\mathrm{NC}}$-values to the nitrogen enrichment expected from mass transfer in Algol and nitrogen dredge-up in $\beta$ Cet and suggest that also in the cases of HR 1099 and UX Ari CNO-cycle burning with ensuing nitrogen dredge-up has begun.

If we accept a value of $R_{\mathrm{NC}}=0.57$ as the maximally possible one for a cosmic abundance plasma, the

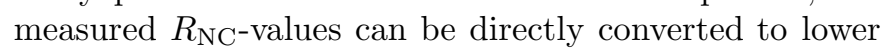
limits for the deviations of the $\mathrm{N} / \mathrm{C}$ abundance with respect to cosmic abundances. We note that these values are independent of the actually prevailing differential emission measure distribution. We specifically find for Algol $\log \Delta \mathrm{N} / \mathrm{C}>1.61$, for $\beta$ Cet $\log \Delta \mathrm{N} / \mathrm{C}>1.17$, while for UX Ari we find $\log \Delta \mathrm{N} / \mathrm{C}>0.55$ and for $\mathrm{HR} 1099$ $\log \Delta \mathrm{N} / \mathrm{C}>0.16$.

How reliable are those numbers? In Table 1 we quote the statistical measurement errors. In order to obtain flux ratios, one has to multiply with the ratio of the effective LETGS areas at $24.74 \AA$ and $33.74 \AA$, i.e., only the relative error enters, which is expected to be $<5 \%$. The theoretical $R_{\mathrm{NC}}$ ratio depends on the collisional excitation rates and the ionization equilibrium. These errors are difficult to specify; the former are believed to have accuracies of $\approx 20 \%$. As far as the latter are concerned, different equilibrium calculations may result in a small shift in temperature scale. We therefore conclude that the deviations of the observed $R_{\mathrm{NC}}$ ratio for HR 1099, UX Ari, and specifically of $\beta$ Cet and Algol cannot be attributed to errors in the model, but have a physical cause.

How unique is our proposed interpretation? Can we be sure that we actually do see the late-type star in the Algol system? While a mathematical proof for the absence of X-ray emission from Algol A cannot be given, such an assumption appears extremely unlikely. First, none of the single A-type stars in the solar neighborhood are X-ray sources (cf. Schmitt 1997). Second, in the case of the eclipsing binary $\alpha \mathrm{CrB}$, consisting of a primary of spectral type A0V and a secondary of type G2V, a total X-ray eclipse is observed, demonstrating that the early-type star can contribute a minute fraction of the total X-ray flux at best (Schmitt \& Kürster 1993). And third, a long duration flare observed on Algol with BeppoSAX (Schmitt \& Favata 1999) shows a total eclipse of the flare plasma at (optical) secondary minimum, when Algol A is seen in front of Algol B, thus demonstrating that at least the flare was associated with Algol B. We therefore conclude that it is extremely unlikely that Algol A significantly contributes to the total X-ray flux from this system.

In summary, we have shown that the LETGS spectra of Algol, $\beta$ Cet, and the RS CVn binaries Capella, HR 1099, and UX Ari can only be understood by an enhanced nitrogen abundance. It is natural to attribute this enhancement to the CNO-cycle operating in the cores of these stars. X-ray spectroscopy can therefore be used to test the predictions of stellar evolution theory.

Acknowledgements. J.-U.N. acknowledges financial support from Deutsches Zentrum für Luft- und Raumfahrt e.V. (DLR) under 50OR98010. Fruitful discussions with Prof. H. Kähler on stellar evolution are acknowledged.

\section{References}

Al-Naimiy, H. M. K., Mutter, A. A. A., \& Flaih, H. A. 1985, Ap\&SS, 108, 227

Ayres, T. R., Fleming, T. A., \& Schmitt, J. H. M. M. 1991, ApJ, 376, L45

Böhm-Vitense, E., \& Mena-Werth, J. 1992, ApJ, 390, 253

Crawford, J. A. 1955, ApJ, 121, 71

Goodricke, J. 1783, Phil. Trans. R. Soc. London, 73, 474

Iben, I. Jr. 1967, ARA\&A, 5, 571

Luck, R. E. 1978, ApJ, 219, L148

Luck, R. E., \& Lambert, D. L. 1981, ApJ, 245, 1018

Luck, R. E., \& Lambert, D. L. 1985, ApJ, 298, 782

McCluskey, G. E., \& Kondo, Y. 1992, in The realm of interacting binary stars, ed. J. Sahade, G. E. McCluskey, \& Y. Kondo (Kluwer Academic Publishers), 381

Mewe, R., Gronenschild, E. H. B. M., \& van den Oord, G. H. J. 1985, A\&AS, 62, 197

Ness, J.-U., Mewe, R., Schmitt, J. H. M. M., et al. 2001, A\&A, 367,282

Ness, J.-U., Schmitt, J. H. M. M., Burwitz, V., Mewe, R., \& Predehl, P. 2002, A\&A, 387, 1032

Parthasarathy, M., Lambert, D. L., \& Tomkin, J. 1983, MNRAS, 203, 1063

Pease, D. O., Drake, J. J., Johnson, C. O., et al. 2000, SPIE, 4012, 700 Effective areas for the LETGS measured Oct. 31, 2000

Predehl, P., Bräuninger, H., Brinkman, A. C., et al. 1997, Proc. SPIE, 3113, 172

Schmitt, J. H. M. M., \& Kürster, M. 1993, Science, 262, 215

Schmitt, J. H. M. M. 1997, A\&A, 318, 215

Schmitt, J. H. M. M., \& Favata, F. 1999, Nature, 401, 44

Schröder, K.-P., Hünsch, M., \& Schmitt, J. H. M. M. 1998, A\&A, 335, 591

Sneden, C., Pilachowski, C. A., \& Vandenberg, D. A. 1986, ApJ, 311, 826

Stelzer, B., et al. 2002, A\&A, submitted 